\title{
Violencia escolar en estudiantes de nivel medio superior
}

\section{School violence in high school students}

\author{
Dulce Vanessa Garzón González \\ ORCID: 0000-0003-3574-2031/garzonvanessa80@gmail.com \\ Doctorado en Ciencias Sociales, Facultad de Estudios Superiores de Cuautla (FESC), Universidad Autónoma del Estado de Morelos (UAEM)
}

\section{Miriam de la Cruz Reyes}

ORCID: 0000-0002-6100-5433/miriam.cruz@uaem.mx

Profesora-investigadora, Centro de Investigación Interdisciplinar para el Desarrollo Universitario (CIIDU), UAEM

\section{Juan Salvador Nambo de los Santos}

ORCID: 0000-0002-3891-5228/salvadornambo@gmail.com

Profesor de Asignatura, Facultad de Derecho y Ciencias Sociales (FDYCS), UAEM

\section{RESUMEN}

Este artículo trata sobre la violencia escolar que se manifiesta en adolescentes de las escuelas preparatorias que pertenecen a la Universidad Autónoma del Estado de Morelos (UAEM). Para el análisis se utiliza el Modelo Ecológico del Desarrollo Humano de Urie Bronfenbrenner, el cual da la pauta para comprender la violencia en un nivel micro, que se configura de los aspectos personales, mientras que el nivel meso tiene sentido con las cuestiones familiares-escolares. Se trata de un estudio empírico cuantitativo con metodología selectiva de encuesta, que, mediante un procedimiento estadístico multivariado, analiza la conducta violenta en la escuela, niveles de victimización, satisfacción con la vida, ideación suicida, comunicación familiary percepción de los orientadores escolares.

PALABRAS CLAVE

violencia escolar, adolescencia, modelo ecológico, educación media superior
ABSTRACT

This article is about the school violence that manifests in adolescents from the high schools of Universidad Autónoma del Estado de Morelos (UAEM). The Ecological Model of Human Development by Urie Bronfenbrenner is used for the analysis, which gives the guideline to understand violence at a microlevel, which is shaped from personal aspects, while the meso-level makes sense with family-school issues. This is a quantitative empirical study with a selective survey methodology, which, through a multivaried statistical procedure, analyzes violent behavior at school, levels of victimization, satisfaction with life, suicidal ideation, family communication and the perception of school counselors.

\section{KEY WORDS}

school violence, adolescence, ecological model, high school education 


\section{Introducción'}

De acuerdo con el Fondo de las Naciones Unidas para la Infancia (UNICEF), la violencia tiene consecuencias en las vidas de los más jóvenes: para su salud emocional, su capacidad de aprender e interrelacionarse con otros y su posibilidad de crecer y desarrollarse plenamente, lo que a su vez trae consecuencias muy profundas también para el país (Naciones Unidas en México [ONU México], 2020).

En México, considerado como el país con el mayor número de ciudades violentas (Consejo Ciudadano para la Seguridad Pública y Judicial Penal AC, 2021), las formas de violencia que se presentan en el ámbito social se ven reflejadas en las interacciones que se generan en las instituciones, y las instituciones educativas no son la excepción. El ejemplo está en los resultados del Informe sobre la Educación Obligatoria en México 2018, donde se muestra que los estudiantes de educación media superior manifiestan haber padecido algún tipo de violencia, agresión verbal con el más alto porcentaje, seguido de robo, difamación y agresión física (Instituto Nacional para la Evaluación de la Educación [INEE], 2018).

Saucedo Ramos y Guzmán (2018) analizan las principales tendencias de investigación sobre la violencia escolar en México que se ha desarrollado desde 2002, y consideran que éste ha sido un campo en crecimiento. Las investigaciones que analizan estas autoras destacan la violencia escolar como un problema grave, frecuente y que se incrementa. Asimismo, enfatizan que la violencia escolar es un problema que tiene su origen en las condiciones económicas, sociales, culturales y de organización de las escuelas; por ello es difícil referirse a una noción de violencia escolar unificada. Para estas investigadoras, es necesario identificar las formas de conceptualizar la violencia sin criminalizar la conducta de los alumnos. Otro aspecto que destacan es que la violencia escolar es un concepto polisémico, multidimensional y complejo, por lo que no hay acuerdo entre los investigadores acerca de cómo definirlo.

En otros estudios se pone de manifiesto que la violencia en el ámbito escolar es un problema compartido por la mayor parte de los países del mundo (Olweus, 2005; Romera et al., 2011). En la última década, han sido muchos los esfuerzos e iniciativas nacionales e internacionales que se han llevado a cabo para reducir y prevenir los problemas de bullying y fomentar la convivencia e integración social de los alumnos en el aula (Álvarez-García et al., 2007). La importancia de las iniciativas se deriva de las graves consecuencias que el bullying tiene para el desarrollo y ajuste psicosocial de la víctima y del agresor (Cava et al., 2010).

Asimismo, diversos autores enfatizan que ciertas características del sistema familiar parecen asociarse en gran medida con la manifestación de problemas conductuales en los hijos (Cava et

\footnotetext{
'Esta investigación ha sido elaborada en el marco del Proyecto PRODEP DSA/103 5/14/7513, titulado "Bullying y ciberbullying en adolescentes escolarizados en el estado de Morelos", y su exposición más amplia se encuentra en la tesis titulada Representaciones y prácticas de violencia escolar de alumnos y alumnas de preparatorias del estado de Morelos. Un análisis desde las interacciones familiares, escolares y comunitarias (Garzón, 2019).
}

Año 17, núm. 41, marzo 2021

ISSN: 2007-1760 (impreso) 2448-9026 (digital) | DOI: 10.30973/inventio/2021.17.41/6 
al., 2010; Povedano et al., 2011). Las explicaciones psicológicas destacan que la probabilidad de que el adolescente se implique en comportamientos antisociales y violentos aumenta cuando el proceso de socialización familiar se ve alterado por factores como una disciplina errática, frecuentes conflictos, utilización de patrones inadecuados de comunicación familiar o falta de cohesión afectiva en la familia (Cava et al., 2010).

Otro factor que se destaca en estas investigaciones es la naturalización de las agresiones: los estudiantes no perciben que los están agrediendo porque, con base en sus experiencias, las prácticas violentas se han incrustado en la cultura escolar y son una manera habitual de convivir en la casa, la calle o la escuela (Del Tronco, 2013; Rodríguez, 2015).

En resumen, las investigaciones señalan que la violencia escolar es un problema que se tiene que seguir investigando por las consecuencias que se derivan en las víctimas y en los agresores. Las socializaciones que se generan en la familia y la naturalización de la violencia repercutirán en su persistencia.

En este trabajo se emplea el término violencia escolar, que, de acuerdo con la Secretaría de Educación Pública [SEP] (2018), se considera como cualquier forma de actividad violenta dentro del marco escolar, la cual incluye a todos los individuos de la comunidad escolar y se expresa de manera deliberada y continua en maltrato físico y psicológico, con el objetivo de someter o asustar a una persona. En este sentido, la violencia escolar se puede presentar de manera manifiesta y relacional (Little et al., 2003). La primera se refiere a comportamientos que implican una confrontación directa hacia otros con la intención de causar daño (empujar, pegar, amenazar, insultar), mientras que la segunda no implica una confrontación directa entre el agresor y la víctima, y se define como aquel acto que se dirige a provocar daño en el círculo de amistades de otra persona, o bien en su percepción de pertenencia a un grupo (exclusión social, rechazo social, difusión de rumores).

Se han implementado diversas estrategias de intervención social para preveniry combatir la conducta violenta en los estudiantes, pero no se ha logrado el impacto deseado. Saucedo Ramos y Guzmán (2018) mencionan que el problema radica en que la intervención social se lleva a cabo sin tomar en cuenta los hallazgos de la investigación; las prácticas de intervención se basan o construyen a partir de diagnósticos superficiales o demasiado puntuales que limitan muchas veces los alcances de sus objetivos; además, la heterogeneidad de contextos escolares y de problemáticas exige un conocimiento puntual, pero también profundo y sistemático.

Dado lo anterior, se consideró pertinente realizar un estudio que permitiera comprender las características particulares en las que se manifiesta la violencia en los adolescentes que se encuentran estudiando el nivel medio superior. Se incluyeron nueve preparatorias pertenecientes a la Universidad Autónoma del Estado de Morelos (UAEM), y mediante la aplicación de cinco instrumentos a 1,110 estudiantes, se indagaron aspectos relacionados con 
la conducta violenta en la escuela, niveles de victimización, satisfacción con la vida, ideación suicida, comunicación familiar y percepción de los orientadores escolares.

\section{Metodología}

Se trata de un estudio empírico cuantitativo en el que se aplicó un análisis multivariado y correlacional, con metodología selectiva de encuesta (Rodríguez et al., 2000). Los instrumentos empleados para el levantamiento de la información fueron: 1) Ficha de identificación, que permitió obtener variables sociodemográficas (edad, sexo, lugar de vivienda composición familiar); 2) Escala de conducta violenta en la escuela, que corresponde a las variables sociales (agresión manifiesta y agresión relacional); 3) Escala de victimización, que concierne a las variables de victimización (victimización relacional, victimización manifiesta física y victimización manifiesta verbal); 4) Escala de satisfacción con la vida, para obtener el índice general de satisfacción con la vida, 5) Cuestionario de comunicación familiar, para obtener las variables familiares (comunicación abierta, comunicación ofensiva y comunicación evitativa), y 6) Cuestionario para orientadores, donde las respuestas a los reactivos eran en escala Likert y en algunas ocasiones abiertas.

El análisis de la violencia en adolescentes requiere examinarla en términos de interacción entre personas y contextos (Díaz-Aguado, 2004). Por ello, se adoptaron tres niveles del Modelo Ecológico del Desarrollo Humano de Bronfenbrenner (1979), porque permite analizar la realidad psicosocial de los adolescentes al tratarse de un sistema en el que se articulan, de forma dinámica, los sistemas orgánico, comportamental y ambiental. Los niveles adoptados son: 1) microsistema: incluye todas aquellas actividades, roles y relaciones interpersonales que la persona experimenta en su entorno inmediato determinado y que lleva al control y manejo de las emociones de las personas; 2 ) mesosistema: se refiere a las interacciones existentes entre los contextos del microsistema, como la comunicación entre la familia y la escuela, y 3 ) macrosistema: se refiere a la cultura y momento histórico-social determinado en el que vive la persona e incluye la ideología y valores dominantes en esa cultura. En cada uno de estos niveles se distribuyeron las variables de los instrumentos y se realizaron correlaciones entre niveles y variables (figura 1).

\section{Selección de participantes}

Para el estudio se consideraron nueve preparatorias que pertenecen a la UAEM y que se encuentran ubicadas en distintos municipios del estado. Se encuestó a 1,110 estudiantes de $1^{\circ}$ y $2^{\circ}$ año, $47 \%$ varones y $53 \%$ mujeres, cuyas edades oscilaban entre 14 y 17 años. El estudio cumplió los valores éticos requeridos en la investigación con seres humanos, además de respetar los principios fundamentales incluidos en la Declaración de Helsinki (Asociación Médica Mundial, 2008), en sus actualizaciones y en las normativas vigentes. 


\section{Figura 1}

\section{Distribución y relación de las variables}

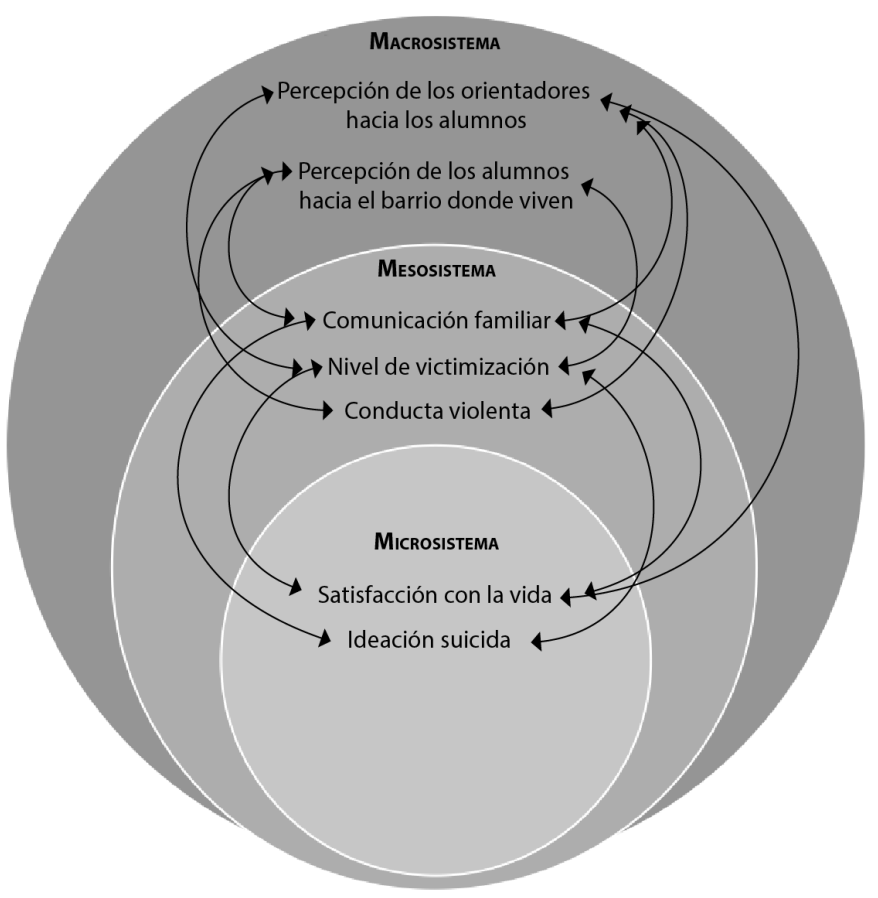

Fuente: Elaboración propia para el análisis de los resultados.

\section{Resultados}

La variable de satisfacción con la vida se considera como un proceso de juicio mediante el cual los individuos valoran la calidad de sus vidas sobre la base de su propio conjunto único de criterios (Pavot y Diener, 1993). En este rubro, los adolescentes contestaron en su mayoría (51.8\%) que están muy en desacuerdo con la frase "no estoy contento con mi vida", lo que significa una relación positiva con la satisfacción de vida; sin embargo, llama la atención que si sumamos las respuestas "de acuerdo" y "muy de acuerdo", se obtiene que el $23.5 \%$ de los estudiantes no están contentos. Las respuestas a la frase "No me gusta todo lo que rodea mi vida", $16.4 \%$ está de acuerdo y 5.9\% muy de acuerdo, lo que revela la insatisfacción que tienen los estudiantes con la vida. El porcentaje más alto de mujeres en el nivel de insatisfacción se encuentra en la Preparatoria 1 (23\%), seguido por Jojutla y Tres Marías (19.5\% y 14.7\%); en el caso de los hombres, el mayor puntaje se encuentra en la preparatoria de Puente de Ixtla (23.8\%), seguido de la Preparatoria 1 y la de Tres Marías, con (18.8\% y 15.1\%).

Con respecto a la ideación suicida correlacionada por edad y sexo, se observa que la frecuencia más alta, 118, se presenta en las mujeres de 16 años, mientras que, en los hombres de la misma edad, se presenta una frecuencia de 71. La ideación suicida en los varones 
disminuye a la edad de 18 años, mientras que en las mujeres se prolonga hasta 19 años. En la Preparatoria 1 se presentaron 68 casos de mujeres y 30 de hombres con ideación suicida, mientras que en las escuelas de Tres Marías, Jojutla y Puente de Ixtla se detectaron 39, 38 y 37 casos, respectivamente.

En cuanto a la comunicación que mantienen los adolescentes con ambos padres, tanto hombres como mujeres manifestaron tener "buena" comunicación, pero al realizar una correlación entre niveles de victimización y comunicación con madre y padre, la presencia de una conducta violenta escolar alta es mayor cuando la comunicación con el padre es poca. Se puede decir que el tipo de comunicación con el padre es una variable primordial. Una constante que se identificó en ambos sexos es que, al presentarse poca comunicación con la madre, existe un alto porcentaje de ideación suicida (hombres, 49.3\%, y mujeres, 44.2\%).

La mayoría de los adolescentes encuestados ha sido víctima de algún tipo de abuso en la escuela: sólo 16\% de los hombres y 17.4\% de mujeres manifestaron no haber sido víctimas. También se encontró una correlación entre el alto nivel de victimización presencial con una alta ideación suicida. Los comportamientos que los alumnos exponen como violencia escolar son: "llamar por apodos", "no hablarle", "reírse cuando se equivocan", "insultarle", "acusarle de cosas que no han dicho o hecho", "contar mentiras acerca de la persona", "burlarse de él por su apariencia física", "imitarlo en forma de burla", "ponerlo en ridículo ante los demás", "decir a otros que no estén con él o que no le hablen", "robar sus cosas" y "agredirlo físicamente".

Una de las preguntas abiertas que se les planteó a los encuestados fue: ¿por qué crees que te molestan? Los hombres consideraron ser molestados porque es una forma de interacción, y dieron respuestas como: "es juego", "así nos llevamos", "por el relajo", "por ser llevados", "por broma", entre otras. Otros mencionaron "mi físico", "orientación sexual", "relaciones amorosas", "formas de pensar diferentes". En cambio, en las mujeres la constante es "envidia", aunque también mencionaron ser molestadas por "el físico", "la condición económica", "ir bien en las materias", "religión", "orientación sexual", entre otros, y en ocasiones se culpaban por permitir ser molestadas o violentadas.

En cuanto al vínculo de los adolescentes con su comunidad, se encontró una relación poco integrada en el caso de las mujeres, ya que no la consideran segura y no cuentan con espacios para ellas, mientras que los hombres manifestaron que sí pueden relacionarse con las personas mayores de su colonia, cuentan con lugares para reunirse con amigos y participan en actividades para jóvenes. Ante la poca seguridad en la colonia, existe un incremento en la victimización de un 42.9\%, comparado con un 46.2\%, mujeres y hombres, respectivamente.

La percepción de los orientadores escolares respecto al agrado o desagrado, y en consecuencias la aceptación, que un alumno logra tener entre sus compañeros, la consideran más alta en los hombres (34.6\%) que en las mujeres (19.9\%). Algo similar ocurre en su percepción respecto a una "buena" relación que mantienen los jóvenes con sus profesores: en el caso de 
los hombres es más alta (61.6\%) y en las mujeres disminuye (41.3\%). A las mujeres se les valoran sus competencias académicas. Además, la implicación familiar que perciben los orientadores es más baja en el caso de los hombres (42.3\%) en comparación con las mujeres (27\%).

\section{Discusión}

Con base en los resultados que se presentan, se da cuenta de que la mayoría de los estudiantes encuestados han sido víctimas de algún tipo de abuso en la escuela; en los hombres es más común que la violencia se presente de forma manifiesta, mientras que en las mujeres es de manera relacional. Para los hombres se consideran determinados actos de violencia como "juego", lo cual tiene similitud con la desviación tolerada de la que habla Dubet (2003), o bien con el deseo del conocimiento del otro (García y Madriaza, 2006). Concebir la violencia de esta manera permite su naturalización y configura el primer momento para que se desarrolle a mayor escala y que sus repercusiones se agudicen, pues ante mayor abuso existen altas probabilidades de que aparezca la ideación suicida.

Se debe considerar el nivel de insatisfacción con la vida que declaran los adolescentes, sobre todo en el caso de las mujeres, porque la insatisfacción tiene una correlación positiva con la ideación suicida, y ésta persiste más tiempo en ellas. En este sentido se requieren estudios que indaguen el origen de ese sentimiento y plantear estrategias para disminuirlo. Los estudiantes identifican aquellos comportamientos que violentan a sus pares, y al llevarlos a cabo estarán avanzado en el desarrollo de los momentos dos y tres de la violencia escolar (García y Madriaza, 2006), al buscar reconocimiento e intentar ascender en una posición jerárquica ante los demás. Sólo en el caso de las mujeres incluso se presenta el sentimiento de culpa por permitir ser violentadas.

Los resultados que se presentan permiten identificar la violencia social (Dubet, 2003), dado que las manifestaciones de conductas violentas están relacionadas con la intolerancia a la diversidad y son recurrentes las prácticas de exclusión a lo diferente, ya sea por desigualdad económica, religión, preferencia sexual o aspectos físicos. Las experiencias de rechazo y el aislamiento social sufridos por los pares de forma reiterada incidirán negativamente en el bienestar psicosocial de niños y adolescentes (Martin y Huebner, 2007).

Un factor que aparece como clave para que se presenten o no conductas violentas o la ideación suicida es la comunicación con ambos padres. Las correlaciones de los datos dan cuenta de que poca interacción con el padre aumenta el índice de victimización, mientras que de la poca comunicación con la madre se incrementa la ideación suicida. Rodrigo et al. (2004) apuntaba que la falta de comunicación o relaciones distantes con los padres pueden favorecer conductas de riesgo en los adolescentes.

Otra forma en que se identifica la violencia social es en la relación que tienen los adolescentes con su comunidad. Las mujeres aparecen como más vulnerables, al no contar con 
personas o espacios en los que se puedan relacionar, mientras que en los hombres se percibe una apropiación de los espacios y mayor relación con los adultos de su colonia.

En cuanto a la violencia antiescuela (Dubet, 2003), la percepción que tienen los orientadores respecto a los estudiantes resulta una forma de violencia por parte de la institución, pues en sus percepciones están presentes diferencias de género que posicionan a las mujeres con menos posibilidades de generar agrado y ser aceptadas por sus compañeros o profesores. Asimismo, perciben diferencias entre las capacidades académicas, pero quedan en mejor valoración las mujeres. El impacto de las percepciones que los maestros tienen hacia los estudiantes es un aspecto que requiere profundizarse.

\section{A manera de cierre}

La violencia escolar es un problema amplio que requiere de un mayor análisis y profundización para obtener elementos que ayuden en el diseño e implementación de estrategias eficientes que lo aminoren. Sin duda, las estrategias tienen que involucrar a los adolescentes, la familia, la escuela e incluso las autoridades de las localidades. Recordemos que mientras esté presente la violencia escolar se impedirá el normal desarrollo de la enseñanza y de las relaciones interpersonales en la convivencia de profesores y alumnos, y de éstos entre sí.

La corresponsabilidad de las instituciones educativas es alta. Antes de criminalizar la conducta de los estudiantes, se tiene que comenzar por analizar las formas en que interactúan las autoridades educativas con ellos e incluso con los padres de familia. La escuela tiene que ofrecer respuestas frente a las nuevas demandas sociales, ofrecer figuras de identificación, además de socializar contenidos que propicien momentos de desestabilización en los que reflexionen sobre sus modos de ser y de convivir, porque los jóvenes merecen desenvolverse en ambientes en los que se sientan seguros.

\section{Referencias}

Álvarez-García, D., Álvarez, L. y Núñez, J. C. (2007). Aprende a resolver conflictos: Programa para mejorar la convivencia escolar. CEPE.

Asociación Médica Mundial (2008). Declaración de Helsinki de la Asociación Médica Mundial. Principios éticos para las investigaciones médicas en seres humanos. Anales del Sistema Sanitario de Navarra. https://www.wma.net/es/policies-post/declaracion-de-helsinkide-la-amm-principios-eticos-para-las-investigaciones-medicas-en-seres-humanos/

Bronfenbrenner, U. (1979). La ecología del desarrollo humano. Paidós Ibérica.

Cava, M. J., Buelga, S., Musitu, G. y Murgui, S. (2010). Violencia escolar entre adolescentes y sus implicaciones en el ajuste psicosocial: un estudio longitudinal. Revista de Psicodidáctica, 15 (1), 21-34. https://ojs.ehu.eus/index.php/psicodidactica/article/view/732 
Consejo Ciudadano para la Seguridad Pública y Judicial Penal AC (2021). Metodología del ranking 2020 de las 50 ciudades más violentas del mundo. http://www.seguridadjusticiaypaz. org.mx/sala-de-prensa/1597-metodologi-a-del-ranking-2020-de-las-50-ciudades-mas-violentas-del-mundo

Del Tronco, J. (2013). La violencia en las escuelas secundarias de México. Una exploración de sus dimensiones. FLACSO.

Díaz-Aguado, M. J. (2004). La violencia entre iguales en la escuela y en el ocio. Estudios comparativos e instrumentos de evaluación. Prevención de la violencia y lucha contra la exclusión desde la adolescencia. INJUVE. http://www.injuve.es/conocenos/edicionesinjuve/materiales-prevencion-de-la-violencia-y-lucha-contra-la-exclusion-desde-laadolescencia-volumen 1

Dubet, F. (2003). Las figuras de la violencia en la escuela. Docencia, 9, 27-37. https://www. revistadocencia.cl/web/index.php/ediciones-anteriores/25-docencia19

García, M. y Madriaza, P. (2006). Estudio cualitativo de los determinantes de la violencia escolar en Chile. Estudios de Psicología, 11 (3), 247-256. https://doi.org/10.159o/S1413294X2006000300001

Garzón, D. (2019). Representaciones y prácticas de violencia escolar de alumnos y alumnas de preparatorias del estado de Morelos. Un análisis desde las interacciones familiares, escolares y comunitarias. UAEM. http://riaa.uaem.mx/xmlui/handle/20.500.12055/1049

Instituto Nacional para la Evaluación de la Educación (2018). Informe La educación obligatoria en México. Informe 2018. http://www.inee.edu.mx/portalweb/informe2018/04 informe/ index.html

Little, T., Henrich, C., Jones, S. y Hawley, P. (2003). Disentangling the "whys" from the "whats" of aggressive behavior. International Journal of Behavioral Development, 27 (2), 122-133. https://doi.org/10.1080/01650250244000128

Martin, K. M. y Huebner, E. S. (2007). Peer victimization and prosocial experiences and emotional well-being of middle school students. Psychology in the Schools, 44 (2), 199208. https://doi.org/10.1002/pits.20216

Naciones Unidas en México (18 de mayo de 2020). UNICEF urge reforzar la protección infantil en México ante el incremento de la violencia contra la infancia. https://www.onu.org. mx/unicef-urge-reforzar-la-proteccion-infantil-en-mexico-ante-el-incremento-de-laviolencia-contra-la-infancia

Olweus, D. (2005). Bullying en la escuela: Datos e intervención. En J. San Martín (Ed.), Violencia y escuela (pp. 13-30). Centro Reina Sofía para el Estudio de la Violencia.

Pavot, W.y Diener, E. (1993). Review of the Satisfaction with Life Scale. Psychological Assessment, 5 (2), 164-172. https://doi.org/10.1037/1040-3590.5.2.164

Año 17, núm. 41, marzo 2021

ISSN: 2007-1760 (impreso) 2448-9026 (digital) | DOI: 10.30973/inventio/2021.17.41/6 
Povedano, A., Hendry, L. B., Ramos, M. J. y Varela, R. (2011). Victimización escolar: clima familiar, autoestima y satisfacción con la vida desde una perspectiva de género. Psychosocial Intervention, 20 (1), 5-12. https://doi.org/10.5093/in2011V2on1a1

Rodríguez, M. R., Martínez, C. R. y Chacón, M. S. (2000). Fundamentos metodológicos en psicología y ciencias afines (pp. 179-194). Pirámide.

Rodrigo, M. J., Máiquez, M. L., García, M., Mendoza, R., Rubio, A., Martínez, A. y Martín, J. C. (2004). Relaciones padres-hijos y estilos de vida en la adolescencia. Psicothema, 16 (2), 203-210. https://psycnet.apa.org/record/2004-13889-005

Rodríguez, H. M. (2015). La violencia escolar en el bachillerato. Un estudio de caso. Caleidoscopio, 33, 15-43. https://revistas.uaa.mx/index.php/caleidoscopio/article/view/216

Romera, E. M., Del Rey, R.y Ortega, R. (2011). Factores asociados a la implicación en bullying:Un estudio en Nicaragua. Psychosocial Intervention, 20 (2),161-170. https://doi.org/10.5093/ in2o11V2on2a4

Saucedo Ramos, C. L. y Guzmán, C. (2018). La investigación sobre la violencia escolar en México: tendencias, tensiones y desafíos. Revista Cultura y Representaciones Sociales, 12 (24). https://doi.org/10.28965/2018-024-08

Secretaría de Educación Publica (2018). Documento base del Programa Nacional de Convivencia Escolar para autonomía curricular ciclo 2018-2019. https://www.gob.mx/cms/uploads/ attachment/file/333283/DOCUMENTO BASE DEL PROGRAMA NACIONAL DE CONVIVENCIA ESCOLAR PARA AUTONOMIA CURRICULAR 2018 2019.pdf 